\title{
A framework integrating plant growth with hormones and nutrients
}

\author{
Gabriel Krouk ${ }^{1,2}$, Sandrine Ruffel ${ }^{1,2}$, Rodrigo A. Gutiérrez ${ }^{3}$, Alain Gojon ${ }^{2}$, \\ Nigel M. Crawford ${ }^{4}$, Gloria M. Coruzzi ${ }^{1}$ and Benoît Lacombe ${ }^{2}$

\footnotetext{
${ }^{1}$ Center for Genomics and Systems Biology, New York University, 12 Waverly Place, New York, NY 10003, USA

${ }^{2}$ Biochimie et Physiologie Moléculaire des Plantes, CNRS UMR5004/INRA UMR0386/SupAgro/UM2, Montpellier cedex 1, France

${ }^{3}$ Departamento de Genética Molecular y Microbiología, Pontificia Universidad Católica de Chile, Santiago 8331010, Chile

${ }^{4}$ Section of Cell and Developmental Biology, Division of Biological Sciences, University of California at San Diego, La Jolla, CA 92093-0116, USA
}

\begin{abstract}
It is well known that nutrient availability controls plant development. Moreover, plant development is finely tuned by a myriad of hormonal signals. Thus, it is not surprising to see increasing evidence of coordination between nutritional and hormonal signaling. In this opinion article, we discuss how nitrogen signals control the hormonal status of plants and how hormonal signals interplay with nitrogen nutrition. We further expand the discussion to include other nutrient-hormone pairs. We propose that nutrition and growth are linked by a multilevel, feed-forward cycle that regulates plant growth, development and metabolism via dedicated signaling pathways that mediate nutrient and hormonal regulation. We believe this model will provide a useful concept for past and future research in this field.
\end{abstract}

\section{Growth and nutrition connections}

Many organisms use developmental plasticity as a means to face and adapt to fluctuating environmental conditions $[1,2]$. This is particularly true for sessile organisms, which are not able to escape from adverse environmental conditions. In the case of plants, they need to acquire nutrients from the soil in their nearby environment to complete their developmental cycle [3,4]. Modification of growth is a major process that plants use to successfully complete their lifecycle in such a heterogeneous environment, even and especially up to their reproduction phase [5]. This adaptation process is so important that we hypothesize it has contributed to the evolution of dedicated signaling pathways that interconnect nutrient sensing and hormonal signaling in plants. Indeed, a slow-growing plant will need less nutrients in a particular time-frame compared to a fast-growing plant, because the demand for nutrients is correlated with dry weight production. For example in the case of nitrogen $(\mathrm{N})$, if a plant has a growth rate of $1 \mathrm{~g}$ of fresh weight per day, knowing that $0.1-0.5 \%$ of its fresh weight ( 1-5\% of dry weight) is composed of $\mathrm{N}$ (Molecular Weight $=14$ g. $\mathrm{mol}^{-1}$ ), then $\sim 70-350 \mu \mathrm{mol}$ a day of $\mathrm{N}$ will be necessary to obtain optimal growth. This knowledge has already had practical implications for plant physiology and agriculture [6-8], but can now be explored at a more

Corresponding authors: Krouk, G.(gk40@nyu.edu); Lacombe, B. (benoit.lacombe@supagro.inra.fr). molecular level. Indeed, a challenging nutrient-deprived environment will slow growth, which in turn will modify intrinsic nutrient uptake. However, is this purely an effect of the 'law of mass action'? I.e. are the nutrient needs of the plant only conditioned by the sink created by growth, or does this phenomenon use specific, molecularly-defined signaling pathways?

In fact, the existence of such signaling pathways is revealed physiologically by the following observations. It has been demonstrated that the $\mathrm{N}$ content of a plant does not need to be modified in order for one to observe a growth modification. For instance, in tobacco (Nicotiana tabacum), switching from nitrate $\left(\mathrm{NO}_{3}{ }^{-}\right)$to pure ammonium $\left(\mathrm{NH}_{4}{ }^{+}\right)$ nutrition (a mostly less efficient $\mathrm{N}$ source on its own) quickly triggered (within hours) a decrease in shoot growth, even before the $\mathrm{N}$ content of the shoot was modified [9]. This demonstrates that growth is tightly controlled in plants by nutritional cues, and that it is not, at least in the first hours of the environmental changes, the consequence of a modification of the overall nutritional status (e.g. by changes in the composition of $\mathrm{N}$-containing compounds in the plant).

In this opinion article, we highlight evidence in the literature that supports the existence of a dedicated multi-layer feed-forward loop (Figure 1) that interconnects nutrition and growth signaling, using $\mathrm{N}$ nutrition as an example. We discuss in the first part, how hormonal status is influenced by $\mathrm{N}$-availability, and in the second part, how hormones control N-sensing, -uptake and -assimilation. Finally, we briefly apply this principle to connections between other nutrients and hormones and draw some general conclusions for the consequences of this hypothesized feed-forward loop for the field of hormone and nutrient signaling.

When $\mathbf{N}$ supply modifies the hormonal status of plants $\mathrm{N}$ is a macronutrient present in many key biological molecules and therefore constitutes a limiting factor in agricultural systems [8]. It is available for plants predominantly as $\mathrm{NO}_{3}{ }^{-}$and $\mathrm{NH}_{4}{ }^{+}$. It controls many aspects of plant life, and has a strong impact on plant development (reviewed in [10-12]). $\mathrm{NO}_{3}{ }^{-}$itself has been shown to be a key signal molecule, and the respective effects of the ion and of its downstream metabolites can be separated genetically, in a 


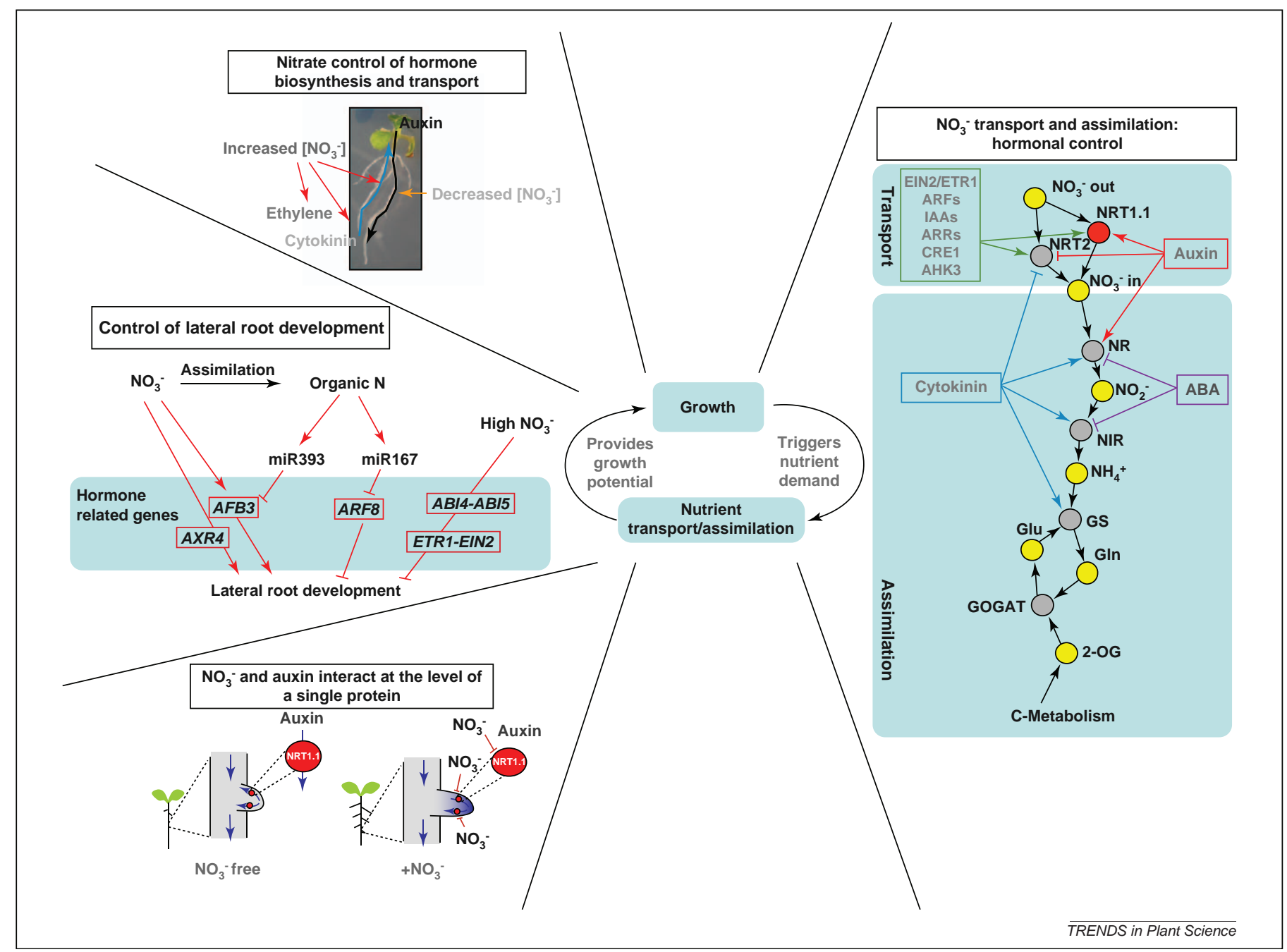

Figure 1. Conceptual model integrating nutrient and hormonal signaling. We hypothesize the existence of a multi-scale (from molecular to physiological interactions) feedforward cycle that interconnects nutrient and hormonal signals. On one hand, nutrients provide growth potential to the plant. On the other hand, growth creates a nutrient demand (central circle of the figure). We propose that this connection is integrated via dedicated signaling pathways that underline the pure 'law of mass action'. This multiscale signaling pathway has begun to be unified and is presented in the different panels (corresponding to different level of integration) of the figure for the nitrogen and

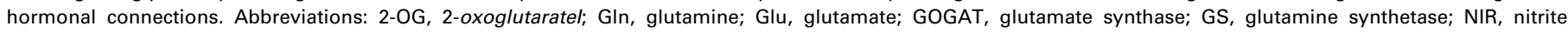
reductase; NR, nitrate reductase.

$\mathrm{NO}_{3}{ }^{-}$reductase double-mutant unable to reduce $\mathrm{NO}_{3}{ }^{-}$into $\mathrm{NH}_{4}^{+}$and therefore into downstream amino acids [13]. In this review, we will use the terms ' $\mathrm{NO}_{3}{ }^{-}$' and ' $\mathrm{N}$-metabolites' as distinct terms to distinguish between the effect of the ion itself or those effects due to the nutritional status of the plant, respectively. When no distinction can be made according to the reviewed data, we will use ' $\mathrm{N}$ ' as a default term, knowing that the distinction remains to be experimentally tested.

The question of how $\mathrm{N}$ supply modifies the hormonal status of plants was addressed early in the history of plant physiology by George S. Avery, Jr. et al., using Brassica caulorapa in the $1940 \mathrm{~s}$. The authors showed that 'extractable' auxin was barely detectable in the tips of stems of Nstarved plants [14]. In a second publication [15], George S. Avery, Jr. and Louise Pottorf determined that there is a significant correlation between $\mathrm{NO}_{3}{ }^{-}$and auxin content in leaves, for plants grown under different $\mathrm{NO}_{3}{ }^{-}$regimes. A ten-fold change in $\mathrm{NO}_{3}{ }^{-}$supply induces a four-fold change in auxin concentration. For high $\mathrm{NO}_{3}{ }^{-}$concentrations, the auxin levels tend to plateau. More recently, modification in auxin content under various $\mathrm{N}$ treatments was studied in soybean (Glycine max) [16], wheat (Triticum aestivum) [17], pineapple (Ananas comosus) [18], maize (Zea mays) [19] and Arabidopsis (Arabidopsis thaliana) [20,21]. In each case, auxin seems to be translocated from shoot-toroot in response to a decrease in $\mathrm{NO}_{3}{ }^{-}$supply (Figure 1 ). Furthermore, genetic modifications in $\mathrm{NO}_{3}{ }^{-}$transporters also affect the hormonal status of the plant. Indeed, a mutant in the high-affinity root $\mathrm{NO}_{3}{ }^{-}$-uptake transporter (lin1/nrt2.1) was reported to display modified auxin accumulation in hypocotyls [22]. More recently, another $\mathrm{NO}_{3}{ }^{-}$ transporter (CHL1/NRT1.1) has been shown to control auxin accumulation in lateral root tips in response to $\mathrm{NO}_{3}{ }^{-}$supply [20]. Indeed, $\mathrm{NO}_{3}{ }^{-}$-dependent hormone transport has been demonstrated at the level of a single protein in both heterologous and homologous systems. NRT1.1 [23], has been demonstrated to be involved in a $\mathrm{NO}_{3}{ }^{-}$-repressed auxin transport (Figure 1). When $\mathrm{NO}_{3}{ }^{-}$ concentration in the media is below $0.2 \mathrm{mM}$, the NRT1.1 protein behaves like an auxin transporter. These observations have led to the hypothesis that the NRT1.1 transporter couples the auxin and $\mathrm{NO}_{3}{ }^{-}$signals through their molecular interaction (Figure 1). In this system, the 
absence of $\mathrm{NO}_{3}{ }^{-}$can be transduced as an increase in auxin flux into the cell and tissue [20].

Similarly, cytokinin content is also under the control of $\mathrm{N}$ supply (for review see [24,25]). For instance, when tomato (Solanum lycopersicum) plants are grown with $\mathrm{NH}_{4}{ }^{+}$as the sole $\mathrm{N}$ source, shoot growth is strongly reduced. In these experiments, $10 \mu \mathrm{M}$ of $\mathrm{NO}_{3}{ }^{-}$is sufficient to restore shoot growth comparable to the growth of $2 \mathrm{mM}$ $\mathrm{NO}_{3}{ }^{-}$-fed plants [26]. These contrasting $\mathrm{N}$-supplies modify $\mathrm{NO}_{3}{ }^{-}$concentrations in xylem exudates, which are consistently associated with an increase in cytokinin concentration [26]. At the molecular level, the Arabidopsis IPT3 gene (an isopentenyl transferase responsible for the limiting step in the biosynthesis of cytokinins) is strongly induced by $\mathrm{NO}_{3}{ }^{-}$in both roots and shoots [13]. The IPT3 gene seems to be a key actor involved in cytokinin status modification in response to $\mathrm{NO}_{3}{ }^{-}$. Indeed, ipt3 knock-out (KO) plants, lacking a functional IPT3 gene, are affected in the $\mathrm{NO}_{3}{ }^{-}$-dependent cytokinin synthesis [27].

Recent investigations have also revealed ethylene as a potential target of $\mathrm{N}$-related signals. Indeed, ethylene production is enhanced in plants transferred from a low $(0.1 \mathrm{mM})$ to a high $(10 \mathrm{mM}) \mathrm{NO}_{3}{ }^{-}$-containing media within one hour of transfer [28].

These observations confirm a tight control of hormone synthesis and transport by $\mathrm{NO}_{3}{ }^{-}$and $\mathrm{N}$ supply. This control leads to profound modifications of plant development. This phenomenon particularly impacts on root architecture [29]. For example, it has been shown that local $\mathrm{NO}_{3}{ }^{-}$stimulates lateral root development (LRD) through auxin remobilization. This was first demonstrated in 2005 in maize [30] by providing TIBA (2,3,5-triiodobenzoic acid, which inhibits auxin transport) between shoot and roots, in order to stop auxin fluxes between these organs. In this instance, $\mathrm{LRD}$ in response to local supply of $\mathrm{NO}_{3}{ }^{-}$, is completely abolished in the presence of TIBA when it is provided in a split-media, between shoots and roots. However, application of TIBA below the site of $\mathrm{NO}_{3}{ }^{-}$application does not affect $\mathrm{NO}_{3}{ }^{-}$-induced LRD [30]. This demonstrates that auxin transport from shoot to root might be a necessary messenger for $\mathrm{NO}_{3}{ }^{-}$presence [29].

Molecular actors involved in auxin transport and signaling are known to be controlled by $\mathrm{NO}_{3}{ }^{-}$and $\mathrm{N}$ provision. This is exemplified by the auxin receptor TIR1 [31], and its homologous gene $A F B 3$, two auxin carriers (At2g17500 and At1g76520), and four efflux auxin transporters (PIN1, PIN2, PIN4 and PIN7) all of which were shown to be controlled at the transcriptional level by carbon and/or $\mathrm{N}$ treatments [32,33]. To date, AFB3 was found to be transiently induced by $\mathrm{NO}_{3}{ }^{-}$in Arabidopsis roots, specifically at the root tip and in pericycle cells. The upregulation of $A F B 3$ correlates with an increased auxin signaling in Arabidopsis roots. Analysis of AFB3 mutants shows that this specific auxin receptor is required for the normal root response to changes in $\mathrm{NO}_{3}{ }^{-}$levels. Interestingly, miR393 (a miRNA targeting F-box proteins such as TIR1 and AFB3) is induced by $\mathrm{N}$-metabolites generated downstream of $\mathrm{NO}_{3}{ }^{-}$reduction. In a miR393 KO plant, AFB3 mRNA level is still early $\mathrm{NO}_{3}{ }^{-}$-induced (within one hour) but is no longer repressed at longer time points (at two- and fourhour treatments). Thus, the miR393:AFB3 regulatory module constitutes an incoherent feed-forward mechanism [34] that can integrate external $\mathrm{NO}_{3}{ }^{-}$availability and the internal $\mathrm{N}$ status of the plant for balanced $\mathrm{N}$-acquisition through a developmental response [33]. Another molecular actor controlling hormone transport is NRT1.1. Indeed, the NRT1.1 gene can be considered as one such target since (i) it controls the auxin flux in the lateral root and (ii) it is controlled by $\mathrm{NO}_{3}{ }^{-}$transcriptionally and at the level of its auxin transport activity [20].

Taken together, these results show that $\mathrm{N}$ in general, and $\mathrm{NO}_{3}{ }^{-}$in particular, regulate plant development at several levels of integration through the control of hormone synthesis, transport and signaling (see Figure 1, left part of the cycle). This constitutes the first half of the conceptual cycle described in Figure 1, where nutrient cues influence hormonal signaling pathways to potentially modify growth. In the second part of the cycle, through hormonal signaling pathways, growth can tweak nutrient acquisition assimilation and perception as described below.

\section{When hormones regulate $\mathbf{N}$ acquisition and assimilation}

Different hormones have been involved in mineral nutrition of plants. Direct effects of these hormones on $\mathrm{N}$ acquisition and $\mathrm{N}$-assimilation genes have been demonstrated. This constitutes a positive retro-control of growth on $\mathrm{N}$ nutrient uptake, and $\mathrm{N}$-assimilation and is very likely supported by dedicated signaling pathways.

It was in the early $1990 \mathrm{~s}$, during the emergence of the field of molecular physiology, that this question was first addressed. $\mathrm{NO}_{3}{ }^{-}$reductase (NR) activity (NRA) was shown to decrease rapidly in excised chicory root (Cichorium intybus L. Witloof cv. Flash), due to an increase in its phosphorylation status [35]. In a second publication, the same group showed that auxin and cytokinin, when supplied before excision, can maintain the level for mRNA, protein and activity for NR. Finally, roots incubated in a $\mathrm{NO}_{3}{ }^{-}$-free medium, before cytokinin treatment, were delayed in their transcriptional activation of NR. This indicates an interaction of the $\mathrm{NO}_{3}{ }^{-}$and cytokinin signaling pathways in the control of NR [36]. Further, cytokinins have been shown to regulate NR mRNA in tobacco cell suspension culture [37], barley (Hordeum vulgare) leaves [38] and Arabidopsis [39].

Two of the main players in $\mathrm{NO}_{3}{ }^{-}$uptake, NRT1.1 and $N R T 2.1$, are known to be hormone-responsive genes. The promoter activity of NRT1.1 is induced by exogenous and endogenous increase of auxin. This induction is independent of the $\mathrm{NO}_{3}{ }^{-}$presence in the culture medium [40]. This demonstrates hormonal control of the $\mathrm{NO}_{3}{ }^{-}$transporter NRT1.1. Because this protein has also been shown to be a $\mathrm{NO}_{3}{ }^{-}$receptor $[20,41]$, this opens the possibility that the hormonal retro-control might target $\mathrm{NO}_{3}{ }^{-}$transport and perception. For NRT2.1 (a major component of the High Affinity $\mathrm{NO}_{3}{ }^{-}$Transport), the NRT2.1 mRNA level strongly decreases within hours of auxin and cytokinin supply. Interestingly, auxin repression can be weakened in detopped plants, suggesting the necessity for the auxin signal to be integrated in the shoot to repress NRT2.1 in the roots, or to interact with a shoot-derived signal [42]. In a genomewide analysis of cytokinin responsive genes, NRT2.1, NRT2.3 and NRT2.6 were found to also be under strong 
cytokinin repression [39]. Recently, these genes (including NRT2.1) and several other $\mathrm{NO}_{3}{ }^{-}$transporters where shown to be gradually regulated by increasing cytokinin concentrations [25]. Furthermore, a meta-analysis of gene expression data has shown that most of the genes involved in $\mathrm{NO}_{3}{ }^{-}$transport and assimilation are controlled (as a group, as defined by bi-clustering) in response to several hormone treatments - predominantly abscisic acid (ABA) and cytokinins [43]. This analysis demonstrates that genes being controlled by both signals $\left(\mathrm{NO}_{3}{ }^{-}\right.$and hormones) are more responsive to $\mathrm{NO}_{3}{ }^{-}$than the genes controlled by $\mathrm{NO}_{3}{ }^{-}$only, and supports the hypothesis that hormones enhance the $\mathrm{NO}_{3}{ }^{-}$response [43].

From a genetic point of view, phenotypic analysis of several KO mutant plants demonstrates a role of the hormone-signaling pathways in the control of $\mathrm{NO}_{3}{ }^{-}$transport, $\mathrm{NO}_{3}{ }^{-}$assimilation and $\mathrm{NO}_{3}{ }^{-}$controlled development.

Concerning controlled development, the response of LRD to N-treatment is partially dependent on auxin-related genes, such as AXR4 [44], ARF8 [45] and AFB3 [33]. Similarly, analysis of ETR1 and EIN2, the ethylene signal transducer genes, have been shown to control N-regulation of lateral root length and the NRT1.1 and NRT2.1 genes [28], themselves involved in LRD [20,46,47]. Furthermore, $\mathrm{ABA}$ related transcription factors $\mathrm{ABI} 4$ and $\mathrm{ABI} 5$, are involved in repression of LRD in response to high $\mathrm{NO}_{3}{ }^{-}$ concentrations [48].

Subsequently, transcriptomic analysis of the axr3 mutant [49] and the arf7; arf19 double mutant [50] (involved in auxin signaling) showed that the NRT2.1 gene is overexpressed in these genetic backgrounds. More recently, the regulation of the $\mathrm{NO}_{3}{ }^{-}$transporters by increasing provision of cytokinin showed reduced sensitivity in cre112;ahk3-3 (a double mutant for cytokinin receptors), and hypersensitivity in arr3; arr4;arr5;arr6; arr8;arr9 (a hextuple mutant for six negative response regulators involved in the cytokinin signaling pathway) [25].

These data support the idea that hormonal signaling pathways (in this case auxin, cytokinins, ethylene and ABA) control plant behavior in response to fluctuating $\mathrm{N}$ environments. Together with the influence of $\mathrm{N}$ on the hormonal status of the plant, this builds the conceptual cycle described in Figure 1, where nutrients control growth, which in turn controls nutrient use and perception.

\section{What about the other nutrients?}

So far we have focused on $\mathrm{N}$ and hormone relationships because they are the best documented to date, especially for the effect of $\mathrm{N}$ on hormone signaling and synthesis. However, we believe that this 'duality of growth' concept as the 'Alpha and Omega' of plant nutrition can be applied to the other nutrients. For instance, cross-talk has been documented for the following nutrient and hormone pairs: sulfate and cytokinins [51], phosphate and cytokinins [52,53], phosphate and auxin [54], potassium and auxin [55], potassium and jasmonic acid [56], iron and cytokinins [57], and phosphate and strigolactones [58,59]; for an excellent general review see [60].

It is noteworthy that for most of the scenarios cited above, these cross-talks concern the role of hormones in the control of genes involved in nutrition, such as transporter or reducing enzymes. We would like to emphasize the fact that most of the recorded effects of the exogenous applied hormones on gene expression failed to identify any interaction with the nutritional signals themselves [40,5153,57]. For instance, transporters involved in the uptake of nutrients from soil, tend to be largely repressed by cytokinins. This cytokinin repression is lost in mutants deficient in cytokinin receptors. However, the transcriptional regulation of the transporters by their substrates (e.g. IRT by iron, and SULTR by sulfate) is not affected in hormone receptor mutants. It is therefore tempting to speculate that hormones (here cytokinins), are a global reporter of the satiety of the plant and control nutrient transport with a low level of specificity. By contrast, we have cases where EIN2 mutation, affecting ethylene-signaling, disrupts transcriptional responses to $\mathrm{NO}_{3}{ }^{-}$[28]. This demonstrates that some hormones might be also involved in the nutrient signaling system themselves. Thus, deciphering the specificity of hormone implication in the control of nutrient sensing and assimilation is a challenging and important task for the next decade.

\section{Conclusion}

Differential growth and development is a tremendous part of plant adaptation processes in a 'competition for nutrients' context [3,5]. Here, we propose a point of view to understand relationships between nutrition and growth that are intrinsically linked at several levels of integration. On one hand, nutrient provision promotes growth and, on the other hand, growth generates 'demand' signals for nutrients. We hypothesize that this feed-forward cycle is a key element of the integration of growth and nutrition and that it is built by dedicated signaling pathways partly involving hormones.

We believe that this concept can help to contextualize many of the past and forthcoming research concerning crosstalk between nutrition and development of plants, from a physiological perspective to a more molecular point of view.

\section{Acknowledgements}

This paper is dedicated to the memory of Professor Claude Grignon who deeply influenced this field [7] and passed away in 2010. Alain Gojon was supported by the Agropolis Foundation (Grant No. 07024). Nigel M. Crawford's work was supported by the National Science Foundation (Grant Nos. IOB-0519985 and MCB-0929338) and the National Institutes of Health (Grant No. GM040672). We acknowledge funding to Gloria M. Coruzzi from National Institutes of Health (NIH) NIGMS Grant GM032877, National Science Foundation (NSF) Arabidopsis 2010 Genome Grant MCB-0929338 and DOE Grant DEFG02-92ER20071. Research in Rodrigo A. Gutiérrez's laboratory is funded by the Fondo Nacional de Desarrollo Científico y Tecnológico (1100698), National Institutes of Health-Fogarty International Research Collaboration Award (F6414-01), Millennium Nucleus for Plant Functional Genomics (P06-009-F) and ANR-CONICYT (07). Gabriel Krouk's research was supported by a European-FP7-International Outgoing Fellowships (Marie Curie) (AtSYSTM-BIOL; PIOF-GA-2008-220157). Benoît Lacombe was supported by CNRS (Programme PEPS/InSB).

\section{References}

1 Meyerowitz, E.M. (2002) Plants compared to animals: the broadest comparative study of development. Science 295, 1482-1485

2 Borges, R.M. (2005) Do plants and animals differ in phenotypic plasticity? J. Biosci. 30, 41-50

3 Schachtman, D.P. and Shin, R. (2007) Nutrient sensing and signaling: NPKS. Annu. Rev. Plant Biol. 58, 47-69 
4 Gojon, A. et al. (2009) Root uptake regulation: a central process for NPS homeostasis in plants. Curr. Opin. Plant Biol. 12, 328-338

5 Walter, A. et al. (2009) Environmental effects on spatial and temporal patterns of leaf and root growth. Annu. Rev. Plant Biol. 60, 279-304

6 Imsande, J. and Touraine, B. (1994) N demand and the regulation of nitrate uptake. Plant Physiol. 105, 3-7

7 Grignon, C. (1990) Transports in roots. Symbiosis 9, 3-17

8 Marschner, A. (1995) Mineral Nutrition of Higher Plants, Academic Press

9 Walch-Liu, P. et al. (2000) Rapid effects of nitrogen form on leaf morphogenesis in tobacco. J. Exp. Bot. 51, 227-237

10 Vidal, E.A. and Gutierrez, R.A. (2008) A systems view of nitrogen nutrient and metabolite responses in Arabidopsis. Curr. Opin. Plant Biol. 11, 521-529

11 Krouk, G. et al. (2010) Nitrate signaling: adaptation to fluctuating environments. Curr. Opin. Plant Biol. 13, 266-273

12 Crawford, N.M. (1995) Nitrate - nutrient and signal for plant-growth. Plant Cell 7, 859-868

13 Wang, R. et al. (2004) Genomic analysis of the nitrate response using a nitrate reductase-null mutant of Arabidopsis. Plant Physiol. 136, 2512-2522

14 Avery, G.S. et al. (1937) Nutrient deficiencies and growth hormone concentration in Helianthus and Nicotiana. Am. J. Bot. 24, 553-557

15 Avery, G.S. and Pottorf, L. (1945) Auxin and nitrogen relationships in green plants. Am. J. Bot. 32, 666-669

16 Caba, J.M. et al. (2000) Inoculation and nitrate alter phytohormone levels in soybean roots: differences between a supernodulating mutant and the wild type. Planta 211, 98-104

17 Chen, J.G. et al. (1998) Involvement of endogenous plant hormones in the effect of mixed nitrogen source on growth and tillering of wheat. $J$. Plant Nutr. 21, 87-97

18 Tamaki, V. and Mercier, H. (2007) Cytokinins and auxin communicate nitrogen availability as long-distance signal molecules in pineapple (Ananas comosus). J. Plant Physiol. 164, 1543-1547

$19 \mathrm{Liu}$, J. et al. (2010) Auxin transport in maize roots in response to localized nitrate supply. Ann. Bot. 106, 1019-1026

20 Krouk, G. et al. (2010) Nitrate and auxin transport by NRT1.1 defines a mechanism for nutrient sensing in plants. Dev. Cell 18, 927-937

21 Walch-Liu, P. et al. (2006) Nitrogen regulation of root branching. Ann. Bot. $97,875-881$

22 Malamy, J.E. (2005) Intrinsic and environmental response pathways that regulate root system architecture. Plant Cell Environ. 28, 67-77

23 Tsay, Y.F. et al. (1993) The herbicide sensitivity gene CHL1 of Arabidopsis encodes a nitrate-inducible nitrate transporter. Cell 72, 705-713

24 Sakakibara, H. et al. (2006) Interactions between nitrogen and cytokinin in the regulation of metabolism and development. Trends Plant Sci. 11, 440-448

25 Kiba, T. et al. (2011) Hormonal control of nitrogen acquisition: roles of auxin, abscisic acid, and cytokinin. J. Exp. Bot. 62, 1399-1409

26 Rahayu, Y.S. et al. (2005) Root-derived cytokinins as long-distance signals for $\mathrm{NO}_{3}{ }^{-}$-induced stimulation of leaf growth. J. Exp. Bot. 56, 1143-1152

27 Takei, K. et al. (2004) AtIPT3 is a key determinant of nitrate-dependent cytokinin biosynthesis in Arabidopsis. Plant Cell Physiol. 45, 1053-1062

28 Tian, Q.Y. et al. (2009) Ethylene is involved in nitrate-dependent root growth and branching in Arabidopsis thaliana. New Phytol. 184, 918-931

29 Forde, B.G. (2002) Local and long-range signaling pathways regulating plant responses to nitrate. Annu. Rev. Plant Biol. 53, 203-224

30 Guo, Y. et al. (2005) Auxin transport from shoot to root is involved in the response of lateral root growth to localized supply of nitrate in maize. Plant Sci. 169, 894-900

31 Dharmasiri, N. et al. (2005) The F-box protein TIR1 is an auxin receptor. Nature 435, 441-445

32 Gutierrez, R.A. et al. (2007) Qualitative network models and genomewide expression data define carbon/nitrogen-responsive molecular machines in Arabidopsis. Genome Biol. 8, R7

33 Vidal, E.A. et al. (2010) Nitrate-responsive miR393/AFB3 regulatory module controls root system architecture in Arabidopsis thaliana. Proc. Natl. Acad. Sci. U.S.A. 107, 4477-4482

34 Mangan, S. and Alon, U. (2003) Structure and function of the feed-forward loop network motif. Proc. Natl. Acad. Sci. U.S.A. 100, 11980-11985

35 Vuylsteker, C. et al. (1997) Nitrate reductase activity in chicory roots following excision. J. Exp. Bot. 48, 59-65
36 Vuylsteker, C. et al. (1997) Influence of BAP and NAA on the expression of nitrate reductase in excised chicory roots. J. Exp. Bot. 48, 1079-1085

$37 \mathrm{Lu}$, J. et al. (1992) Transcriptional regulation of nitrate reductase mRNA levels by cytokinin-abscisic acid interactions in etiolated barley leaves. Plant Physiol. 98, 1255-1260

38 Suty, L. et al. (1993) Cytokinin affects nitrate reductase expression through the modulation of polyadenylation of the nitrate reductase mRNA transcript. Plant Sci. 90, 11-19

39 Brenner, W.G. et al. (2005) Immediate-early and delayed cytokinin response genes of Arabidopsis thaliana identified by genome-wide expression profiling reveal novel cytokinin-sensitive processes and suggest cytokinin action through transcriptional cascades. Plant J. 44, 314-333

40 Guo, F.Q. et al. (2002) The Arabidopsis dual-affinity nitrate transporter gene AtNRT1.1 (CHL1) is regulated by auxin in both shoots and roots. J. Exp. Bot. 53, 835-844

41 Ho, C.H. et al. (2009) CHL1 functions as a nitrate sensor in plants. Cell $138,1184-1194$

42 Gan, Y. et al. (2005) Nutritional regulation of ANR1 and other rootexpressed MADS-box genes in Arabidopsis thaliana. Planta 222, 730742

43 Nero, D. et al. (2009) A system biology approach highlights a hormonal enhancer effect on regulation of genes in a nitrate responsive "biomodule". BMC Syst. Biol. 3, 59

44 Zhang, H. et al. (1999) Dual pathways for regulation of root branching by nitrate. Proc. Natl. Acad. Sci. U.S.A. 96, 6529-6534

45 Gifford, M.L. et al. (2008) Cell-specific nitrogen responses mediate developmental plasticity. Proc. Natl. Acad. Sci. U.S.A. 105, 803-808

46 Remans, T. et al. (2006) The Arabidopsis NRT1.1 transporter participates in the signaling pathway triggering root colonization of nitrate-rich patches. Proc. Natl. Acad. Sci. U.S.A. 103, 19206-19211

47 Little, D.Y. et al. (2005) The putative high-affinity nitrate transporter NRT2.1 represses lateral root initiation in response to nutritional cues. Proc. Natl. Acad. Sci. U.S.A. 102, 13693-13698

48 Signora, L. et al. (2001) ABA plays a central role in mediating the regulatory effects of nitrate on root branching in Arabidopsis. Plant $J$. $28,655-662$

49 Overvoorde, P.J. et al. (2005) Functional genomic analysis of the AUXIN/INDOLE-3-ACETIC ACID gene family members in Arabidopsis thaliana. Plant Cell 17, 3282-3300

50 Okushima, Y. et al. (2005) Functional genomic analysis of the AUXIN RESPONSE FACTOR gene family members in Arabidopsis thaliana: unique and overlapping functions of ARF7 and ARF19. Plant Cell 17, 444-463

51 Maruyama-Nakashita, A. et al. (2004) A novel regulatory pathway of sulfate uptake in Arabidopsis roots: implication of CRE1/WOL/AHK4mediated cytokinin-dependent regulation. Plant J. 38, 779-789

52 Franco-Zorrilla, J.M. et al. (2005) Interaction between phosphatestarvation, sugar, and cytokinin signaling in Arabidopsis and the roles of cytokinin receptors CRE1/AHK4 and AHK3. Plant Physiol. $138,847-857$

53 Franco-Zorrilla, J.M. et al. (2002) Mutations at CRE1 impair cytokinininduced repression of phosphate starvation responses in Arabidopsis. Plant J. 32, 353-360

54 Nacry, P. et al. (2005) A role for auxin redistribution in the responses of the root system architecture to phosphate starvation in Arabidopsis. Plant Physiol. 138, 2061-2074

55 Vicente-Agullo, F. et al. (2004) Potassium carrier TRH1 is required for auxin transport in Arabidopsis roots. Plant J. 40, 523-535

56 Armengaud, P. et al. (2004) The potassium-dependent transcriptome of Arabidopsis reveals a prominent role of jasmonic acid in nutrient signaling. Plant Physiol. 136, 2556-2576

57 Seguela, M. et al. (2008) Cytokinins negatively regulate the root iron uptake machinery in Arabidopsis through a growth-dependent pathway. Plant J. 55, 289-300

58 Umehara, M. et al. (2008) Inhibition of shoot branching by new terpenoid plant hormones. Nature 455, 195-200

59 Kohlen, W. et al. (2011) Strigolactones are transported through the xylem and play a key role in shoot architectural response to phosphate deficiency in non-AM host Arabidopsis thaliana. Plant Physiol. 155, $615-616$

60 Rubio, V. et al. (2009) Plant hormones and nutrient signaling. Plant Mol. Biol. 69, 361-373 